\title{
Adenomas involving the extrahepatic biliary tree are rare but have an aggressive clinical course
}

Authors

Institutions
Kah Poh Loh' ${ }^{1}$, Deborah Nautsch², James Mueller ${ }^{2}$, David Desilets ${ }^{3}$, Vaibhav Mehendiratta ${ }^{3}$

${ }^{1}$ Division of Hematology/Oncology, James P. Wilmot Cancer Institute, University of Rochester/Strong Memorial Hospital, Rochester, NY, USA

${ }^{2}$ Department of Pathology, Baystate Medical Center/Tufts University School of Medicine, Springfield, MA, USA

${ }^{3}$ Division of Gastroenterology, Baystate Medical Center/Tufts University School of Medicine, Springfield, MA, USA submitted 24. April 2015 accepted after revision 6. October 2015

\section{Bibliography}

DOI http://dx.doi.org/

10.1055/s-0041-107897

Published online: 27.11.2015

Endoscopy International Open 2016; 04: E112-E117

(c) Georg Thieme Verlag KG

Stuttgart · New York

E-ISSN 2196-9736

\section{Corresponding author}

\section{Vaibhav Mehendiratta, MD}

Baystate Medical Center

Western Campus of Tufts

University School of Medicine

759 Chestnut Street, S2606

Springfield

MA 01199

USA

Fax: +1-413-794-8828

vaibhavm23@gmail.com
Biliary adenomas that are usually found in surgically removed gallbladders are rare, but can also occur in the extrahepatic biliary tree. We present a case series of extrahepatic bile duct adenomas at our institution, along with a review of the literature. All three patients with extrahepatic biliary adenomas (two in the common bile ducts, one in the hepatic duct) were female with a mean age of 74 years. On initial presentation, none of the patients had obstructive jaundice but two of the three patients had symptoms of biliary origin. Case 1 is an 85-year-old woman with an incidental biliary dilation seen on chest imaging; endoscopic ultrasound revealed a sessile adenomatous polyp in the distal bile duct. The patient refused surgery and presented with occlusive biliary stricture and jaundice 5 months after initial presentation, with cytology confirming malignant progression. Case 2 is a 78-year-old woman with a history of primary sclerosing cholangitis and who presented with cholangitis, and Gramnegative sepsis. A polypoid lesion was seen on imaging in the common hepatic duct and direct

\section{Introduction}

$\nabla$

Biliary adenomas are rare entities that are usually detected incidentally in gallbladders removed for cholelithiasis or chronic cholecystitis. They can also occur anywhere in the extrahepatic biliary tree. There is limited understanding of the malignant potential of adenomas involving the extrahepatic biliary tree, and there are no guidelines for management. The aim of our study was to identify all extrahepatic biliary adenomas diagnosed at our tertiary care institution, and review their management and clinical outcomes. In addition, we present a literature review of published cases of extrahepatic biliary adenoma. cholangioscopy with biopsies confirmed the presence of adenoma with high grade dysplasia. The patient underwent successful total bile duct resection and hepaticojejunostomy but represented 1 year later with diffuse metastatic disease to the bone, liver, and peritoneum. Case 3 is a 61 -yearold woman who presented with symptoms suggestive of gallbladder pathology and was found to have a polypoid bile duct lesion on intraoperative cholangiogram. Endoscopic retrograde cholangioscopy showed an adenomatous polyp with high grade dysplasia involving the distal common bile duct. The patient underwent distal bile duct resection with choledochojejunostomy but presented with jaundice 4 years after surgery. She was found to have adenocarcinoma involving the small bowel in the Roux limb of jejunum and transverse colon. All three patients in our series presented with interval gastrointestinal malignancy and we therefore recommend aggressive surgical intervention and close postoperative surveillance when diagnosis of extrahepatic bile duct adenoma is made.

\section{Methods}

$\checkmark$

We used the pathology database (CoPath) at our institution to identify patients with a diagnosis of biliary adenoma or adenomatous change on biopsy or surgical resection specimens from year 2000 to 2013. Pathology results from 8774 cholecystectomies (with or without bile duct excision) and 1785 bile duct pinch biopsies were reviewed. Twenty-three patients with a biliary adenoma were identified, arising either in the gallbladder (20/23) or the extrahepatic biliary tree (3/23). All gallbladder biliary adenomas were detected incidentally during cholecystectomy for unrelated indications.

Patient's medical records from the three patients with extrahepatic biliary adenomas were reviewed for demographic information, clinical pre- 
sentation, imaging results, operative findings, and surgical pathology results. The study was approved by the institutional review board at Baystate Medical Center, Springfield, MA.

A literature review of published cases of extrahepatic biliary adenoma was performed using MEDLINE database. All identified cases were reviewed and the findings are summarized.

\section{Results}

$\nabla$

\section{Case 1}

An 85-year-old woman with a history of atherosclerotic disease and gallstones was referred to the Gastroenterology outpatient office for evaluation of an incidental finding of biliary dilation up to $19 \mathrm{~mm}$. The patient complained of intermittent abdominal pain but denied nausea, vomiting, jaundice, or weight loss. Her liver function tests (LFTs) were normal. Endoscopic ultrasound revealed a small soft-tissue non-shadowing lesion in the distal common bile duct (CBD) without evidence of a pancreatic head lesion ( $\bullet$ Fig. 1). Endoscopic retrograde cholangiopancreatography (ERCP) showed diffuse dilation of the biliary tree with a fixed filling defect in the distal CBD without focal stricture. Forceps biopsies revealed papillary and cribriform adenomatous epithelium with high grade dysplasia ( $\bullet$ Fig. 2). A biliary stent was not placed due to normal LFTs. The patient was deemed to be a poor surgical candidate for pancreaticoduodenectomy. Five months after initial presentation, the patient represented with jaundice, decreased appetite, weakness, and weight loss, with an obstructive pattern on her LFTs. ERCP showed a 15-mm occlusive stricture in the distal CBD with diffuse proximal biliary dilation; a metal stent was inserted. Brush cytology showed atypical ductal cells suspicious for adenocarcinoma. One year later, she was found to have duodenal ulceration from underlying cholangiocarcinoma with extensive liver metastases.

\section{Case 2}

A 61-year-old woman presented to the hospital with abdominal pain and weakness. She had a medical history of primary sclerosing cholangitis, and idiopathic thrombocytopenic purpura status post-splenectomy, and was on chronic immunosuppression. Laboratory evaluation revealed leukocytosis, and blood cultures returned extended spectrum, B-lactamase-producing Escherichia coli. MRI of the abdomen showed an irregular, polypoid lesion in the common hepatic duct ( $\bullet$ Fig.3). Direct cholangioscopy with multiple biopsies revealed a villous adenoma with extensive high grade dysplasia. Complete endoscopic polypectomy was unsuccessful, therefore she underwent total bile duct resection and Roux-en-Y hepaticojejunostomy. One year after her initial presentation, she presented with left flank pain and back pain. Imaging revealed bone metastases to the L5-S1 vertebral bodies with biopsy showing adenocarcinoma of pancreaticobiliary origin, along with liver metastases and peritoneal carcinomatosis.

\section{Case 3}

A 78-year-old woman with a history of reflux esophagitis presented with symptoms suggestive of gallbladder pathology. She was found to have a polypoid bile duct lesion on intraoperative cholangiogram. ERCP showed an adenomatous polyp with high grade dysplasia involving the distal CBD. The patient underwent distal bile duct resection with choledochojejunostomy. Four years after surgery, she was found to have a large mass in the roux limb of the jejunum causing obstruction of the small bowel

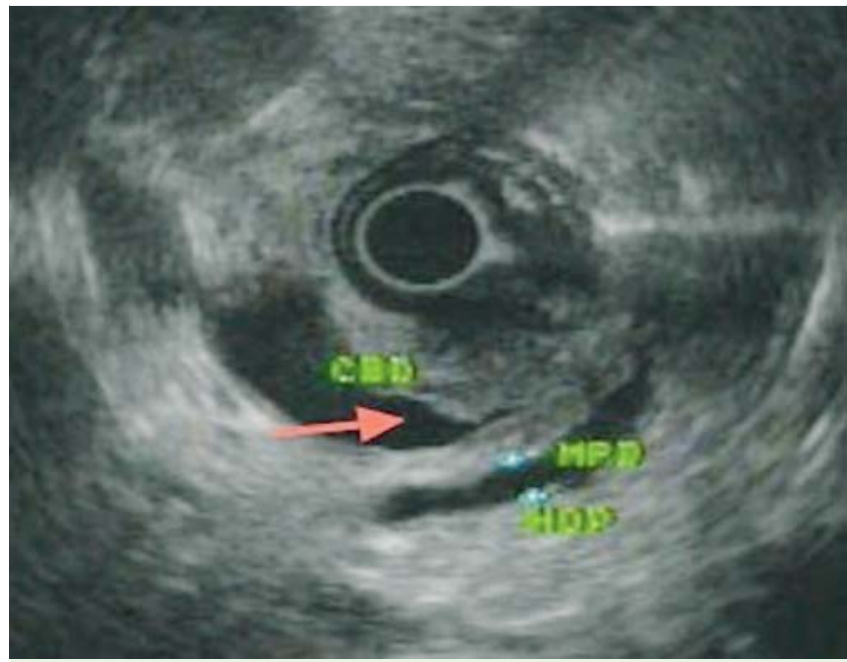

Fig. 1 Endoscopic ultrasound showing non-shadowing lesion in the CBD in the head of the pancreas.

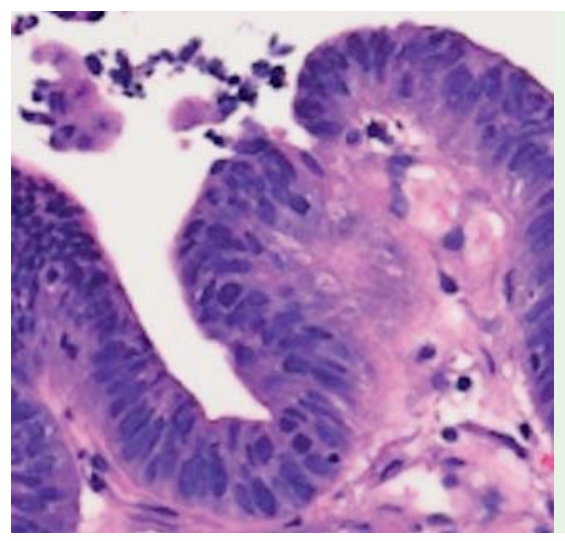

Fig. 2 Forceps biopsy showing adenomatous epithelium with high grade dysplasia.

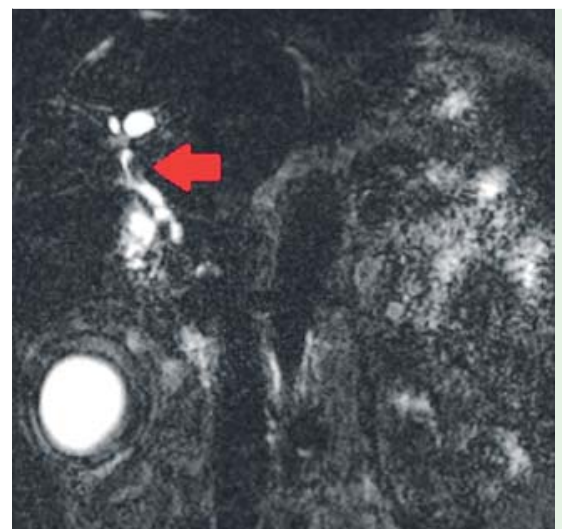

Fig. 3 MRI showing polypoid lesion in the common hepatic duct.

and invading the transverse colon. She underwent transverse colectomy, partial small-bowel resection, resection of the prior hepaticojejunostomy, and creation of a new hepaticojejunostomy. Final pathology showed adenocarcinoma. The patient underwent chemotherapy which was discontinued due to poor tolerance. Two years later, she was found to have metastatic disease to the liver, brain, and skin. 
Table 1 Cases of extrahepatic biliary adenoma reported in the literature including their clinical presentation, histology, treatment, and outcome.

\begin{tabular}{|c|c|c|c|c|c|c|c|c|c|}
\hline Reference & $\mathbf{N}$ & Gender & $\begin{array}{l}\text { Age, } \\
\text { years }\end{array}$ & Country & Location & Presentation & Treatment & Histology & Outcome \\
\hline $\begin{array}{l}\text { Ariche et al. } \\
\text { [2] }\end{array}$ & 1 & $\mathrm{~F}$ & 77 & Israel & Mid CBD & $\begin{array}{l}\text { Recurrent abdom- } \\
\text { inal pain, jaun- } \\
\text { dice, fever }\end{array}$ & $\begin{array}{l}\text { Local excision, roux- } \\
\text { en-y hepatojejunost- } \\
\text { omy }\end{array}$ & $\begin{array}{l}\text { Villous } \\
\text { adenoma }\end{array}$ & - \\
\hline \multirow[t]{2}{*}{$\begin{array}{l}\text { Burhans and } \\
\text { Myers [3] }\end{array}$} & 1 & $\mathrm{~F}$ & 64 & USA & $\begin{array}{l}\text { Left hepatic } \\
\text { duct }\end{array}$ & $\begin{array}{l}\text { Symptoms of cho- } \\
\text { lecystitis, jaun- } \\
\text { dice, fever }\end{array}$ & $\begin{array}{l}\text { Removal with forceps } \\
\text { surgically }\end{array}$ & $\begin{array}{l}\text { Papillary } \\
\text { adenoma }\end{array}$ & $\begin{array}{l}\text { Presented } 4 \text { years later } \\
\text { with large cystic mass. } \\
\text { Alive at } 5 \text { years }\end{array}$ \\
\hline & 1 & $\mathrm{~F}$ & 76 & USA & $\begin{array}{l}\text { CBD (junc- } \\
\text { tion of cys- } \\
\text { tic and bile } \\
\text { duct) }\end{array}$ & $\begin{array}{l}\text { Jaundice, fever, } \\
\text { anorexia, n/v }\end{array}$ & Curettage & Adenoma & $\begin{array}{l}\text { Died } 6 \text { years later from } \\
\text { CVA }\end{array}$ \\
\hline $\begin{array}{l}\text { Hultén et al. } \\
\text { [4] }\end{array}$ & 2 & M & 61 & Sweden & Distal CBD & $\begin{array}{l}\text { Biliary colic and } \\
\text { jaundice }\end{array}$ & $\begin{array}{l}\text { Local excision/chole- } \\
\text { dochectomy and he- } \\
\text { paticoduodenostomy }\end{array}$ & $\begin{array}{l}\text { Papillary } \\
\text { adenoma }\end{array}$ & Alive after 7 years \\
\hline Shemesh [5] & 1 & M & 58 & Israel & Distal CBD & $\begin{array}{l}\text { Recurrent abdom- } \\
\text { inal pain }\end{array}$ & Surgically removed & $\begin{array}{l}\text { Tubular } \\
\text { adenoma }\end{array}$ & Well at 2 months \\
\hline $\begin{array}{l}\text { Sturgis et al. } \\
{[6]}\end{array}$ & 1 & $\mathrm{~F}$ & 81 & UK & Distal CBD & $\begin{array}{l}\text { Intermittent right } \\
\text { upper quadrant } \\
\text { (RUQ) pain, nau- } \\
\text { sea/vomiting }\end{array}$ & Endoscopic excision & $\begin{array}{l}\text { Tubulovil- } \\
\text { lous ade- } \\
\text { noma }\end{array}$ & Well post-surgery \\
\hline $\begin{array}{l}\text { Futami et al. } \\
\text { [7] }\end{array}$ & 1 & $\mathrm{~F}$ & 40 & Japan & $\begin{array}{l}\text { Inferior bile } \\
\text { duct }\end{array}$ & $\begin{array}{l}\text { Relapsing pan- } \\
\text { creatitis }\end{array}$ & Surgical excision & Adenoma & $\begin{array}{l}\text { Uneventful for } 18 \\
\text { months }\end{array}$ \\
\hline Jao et al. [8] & 1 & M & 60 & Taiwan & Distal CBD & $\begin{array}{l}\text { Abdominal } \\
\text { screening ultra- } \\
\text { sound }\end{array}$ & Endoscopic excision & $\begin{array}{l}\text { Tubulovil- } \\
\text { lous ade- } \\
\text { noma }\end{array}$ & Well at 2 months \\
\hline $\begin{array}{l}\text { Ibrarullah } \\
\text { and Sreeni- } \\
\text { vasa [9] }\end{array}$ & 1 & $\mathrm{~F}$ & 33 & India & Distal CBD & $\begin{array}{l}\text { RUQ pain, vomit- } \\
\text { ing }\end{array}$ & $\begin{array}{l}\text { Roux-en-y hepatojeju- } \\
\text { nostomy }\end{array}$ & Adenoma & $\begin{array}{l}\text { Asymptomatic at } 38 \\
\text { months }\end{array}$ \\
\hline $\begin{array}{l}\text { Kim et al. } \\
{[11]}\end{array}$ & 1 & M & 55 & Korea & Distal CBD & $\begin{array}{l}\text { Painless jaundice } \\
\text { and pruritis }\end{array}$ & Whipple & $\begin{array}{l}\text { Tubulovil- } \\
\text { lous ade- } \\
\text { noma }\end{array}$ & $\begin{array}{l}\text { Multiple gastrointesti- } \\
\text { nal polyps } 8 \text { months } \\
\text { after surgery }\end{array}$ \\
\hline $\begin{array}{l}\text { Aparajita et } \\
\text { al. [12] }\end{array}$ & 1 & $\mathrm{~F}$ & 75 & UK & $\begin{array}{l}\text { CBD (junc- } \\
\text { tion at cys- } \\
\text { tic duct) }\end{array}$ & $\begin{array}{l}\text { Jaundice, weight } \\
\text { loss }\end{array}$ & $\begin{array}{l}\text { Pancreaticoduode- } \\
\text { nectomy with Roux- } \\
\text { en-Y reconstruction }\end{array}$ & $\begin{array}{l}\text { Papillary } \\
\text { adenoma }\end{array}$ & $\begin{array}{l}\text { Well } 9 \text { months after } \\
\text { surgery }\end{array}$ \\
\hline $\begin{array}{l}\text { Akaydin et al. } \\
\text { [13] }\end{array}$ & 1 & M & 60 & Turkey & $\begin{array}{l}\text { Proximal } \\
\text { CBD }\end{array}$ & $\begin{array}{l}\text { Painless jaundice, } \\
\text { pruritis, acholic } \\
\text { feces }\end{array}$ & $\begin{array}{l}\text { Excision and Roux-en- } \\
\text { Y hepaticojejunost- } \\
\text { omy }\end{array}$ & $\begin{array}{l}\text { Tubulovil- } \\
\text { lous ade- } \\
\text { noma }\end{array}$ & - \\
\hline $\begin{array}{l}\text { Munshi and } \\
\text { Hassan [14] }\end{array}$ & 1 & $\mathrm{~F}$ & 69 & USA & $\begin{array}{l}\text { Distal CBD, } \\
\text { junction at } \\
\text { cystic duct }\end{array}$ & $\begin{array}{l}\text { RUQ pain, pruritis, } \\
\text { light stools }\end{array}$ & Endoscopic excision & $\begin{array}{l}\text { Papillary } \\
\text { adenoma }\end{array}$ & $\begin{array}{l}\text { Surveillance with no } \\
\text { symptoms, unclear in- } \\
\text { terval }\end{array}$ \\
\hline $\begin{array}{l}\text { Prachayakul } \\
\text { et al. [15] }\end{array}$ & 1 & M & 53 & Thailand & Distal CBD & $\begin{array}{l}\text { Recurrent fever } \\
\text { with intermittent } \\
\text { jaundice }\end{array}$ & $\begin{array}{l}\text { Polypectomy endo- } \\
\text { scopically }\end{array}$ & $\begin{array}{l}\text { Tubular } \\
\text { adenoma }\end{array}$ & $\begin{array}{l}\text { Polyp disappeared on } \\
\text { repeat procedure }\end{array}$ \\
\hline $\begin{array}{l}\text { Sirimonta- } \\
\text { porn et al. } \\
{[16]}\end{array}$ & 1 & M & 73 & Thailand & $\begin{array}{l}\text { Mid to distal } \\
\text { CBD }\end{array}$ & $\begin{array}{l}\text { Recurrent liver } \\
\text { abscess/Klebsiella } \\
\text { bacteremia }\end{array}$ & $\begin{array}{l}\text { Endoscopic forceps } \\
\text { biopsy }\end{array}$ & Adenoma & $\begin{array}{l}\text { Further biopsy normal, } \\
\text { no interventions after- } \\
\text { wards }\end{array}$ \\
\hline $\begin{array}{l}\text { Styne et al. } \\
{[17]}\end{array}$ & 1 & $\mathrm{~F}$ & 59 & USA & $\begin{array}{l}\text { Left hepatic } \\
\text { duct }\end{array}$ & $\begin{array}{l}\text { Recurrent cholan- } \\
\text { gitis }\end{array}$ & Surgical excision & Papilloma & $\begin{array}{l}2 \text { months later adeno- } \\
\text { carcinoma }\end{array}$ \\
\hline $\begin{array}{l}\text { Cardoza et al. } \\
{[18]}\end{array}$ & 1 & $\mathrm{~F}$ & 53 & USA & $\begin{array}{l}\text { Common } \\
\text { hepatic } \\
\text { duct }\end{array}$ & $\begin{array}{l}\text { Incidental LFT ele- } \\
\text { vation }\end{array}$ & Surgical resection & Papilloma & - \\
\hline $\begin{array}{l}\text { Jennings et } \\
\text { al. [19] }\end{array}$ & 1 & M & 58 & UK & $\begin{array}{l}\text { Common } \\
\text { hepatic } \\
\text { duct }\end{array}$ & Jaundice & $\begin{array}{l}\text { Surgically enucleated } \\
\text { and stalk resected }\end{array}$ & $\begin{array}{l}\text { Villous } \\
\text { adenoma }\end{array}$ & $\begin{array}{l}16 \text { months after pre- } \\
\text { sentation, recurrent } \\
\text { villous adenoma, he- } \\
\text { patic duct, roux-en-y }\end{array}$ \\
\hline
\end{tabular}


Table 1 (Continuation)

\begin{tabular}{|c|c|c|c|c|c|c|c|c|c|}
\hline Reference & $\mathbf{N}$ & Gender & $\begin{array}{l}\text { Age, } \\
\text { years }\end{array}$ & Country & Location & Presentation & Treatment & Histology & Outcome \\
\hline $\begin{array}{l}\text { Sotona et al. } \\
\text { [21] }\end{array}$ & 1 & M & 58 & $\begin{array}{l}\text { Czech } \\
\text { Republic }\end{array}$ & $\begin{array}{l}\text { Left hepatic } \\
\text { duct }\end{array}$ & $\begin{array}{l}\text { Painless obstruc- } \\
\text { tive jaundice }\end{array}$ & $\begin{array}{l}\text { Local excision, Roux- } \\
\text { en-Y hepaticojejunost- } \\
\text { omy }\end{array}$ & $\begin{array}{l}\text { Papillary } \\
\text { adenoma }\end{array}$ & $\begin{array}{l}\text { Alive } 1 \text { year after the } \\
\text { surgery }\end{array}$ \\
\hline $\begin{array}{l}\text { Ho and Lee } \\
{[22]}\end{array}$ & 1 & M & 15 & Taiwan & Cystic duct & $\begin{array}{l}\text { Tarry stools, jaun- } \\
\text { dice }\end{array}$ & $\begin{array}{l}\text { Exploratory laparoto- } \\
\text { my }\end{array}$ & $\begin{array}{l}\text { Papillary } \\
\text { adenoma }\end{array}$ & - \\
\hline Loh et al. [23] & 1 & $\mathrm{~F}$ & 72 & UK & Cystic duct & $\begin{array}{l}\text { Recurrent RUQ } \\
\text { pain, nausea }\end{array}$ & $\begin{array}{l}\text { Surgical resection with } \\
\text { cholecystectomy }\end{array}$ & $\begin{array}{l}\text { Papillary } \\
\text { adenoma }\end{array}$ & - \\
\hline Liu et al. [24] & 1 & $\mathrm{~F}$ & 61 & China & Cystic duct & $\begin{array}{l}\text { Intermittent up- } \\
\text { per abdominal } \\
\text { pain and fever }\end{array}$ & $\begin{array}{l}\text { Snare polypectomy } \\
\text { using a gastroscope }\end{array}$ & $\begin{array}{l}\text { Tubulovil- } \\
\text { lous ade- } \\
\text { noma }\end{array}$ & $\begin{array}{l}\text { Asymptomatic at } 3 \\
\text { months }\end{array}$ \\
\hline $\begin{array}{l}\text { O'Shea et al. } \\
\text { [25] }\end{array}$ & 1 & M & 75 & USA & $\begin{array}{l}\text { Left hepatic } \\
\text { and com- } \\
\text { mon hepa- } \\
\text { tic ducts }\end{array}$ & $\begin{array}{l}\text { RUQ pain, jaun- } \\
\text { dice, dark urine, } \\
\text { weakness }\end{array}$ & Excision surgically & $\begin{array}{l}\text { Villous } \\
\text { adenoma }\end{array}$ & - \\
\hline $\begin{array}{l}\text { Morris-Stiff } \\
\text { et al. [26] }\end{array}$ & 1 & $\mathrm{~F}$ & 73 & UK & $\begin{array}{l}\text { Common } \\
\text { hepatic and } \\
\text { proximal } \\
\text { left hepatic } \\
\text { duct }\end{array}$ & $\begin{array}{l}\text { Abdominal pain, } \\
\text { weight loss }\end{array}$ & $\begin{array}{l}\text { Surgical resection, } \\
\text { Roux-en-Y hepaticoje- } \\
\text { junostomy }\end{array}$ & $\begin{array}{l}\text { Papillary } \\
\text { adenoma }\end{array}$ & - \\
\hline $\begin{array}{l}\text { Hanafy and } \\
\text { McDonald } \\
{[27]}\end{array}$ & 1 & M & 76 & UK & $\begin{array}{l}\text { CBD, hepa- } \\
\text { tic and cys- } \\
\text { tic duct }\end{array}$ & $\begin{array}{l}\text { Mild jaundice and } \\
\text { RUQ mass }\end{array}$ & $\begin{array}{l}\text { Local excision surgi- } \\
\text { cally }\end{array}$ & $\begin{array}{l}\text { Villous } \\
\text { adenoma }\end{array}$ & - \\
\hline $\begin{array}{l}\text { Xu and Chen } \\
\text { [28] }\end{array}$ & 1 & $\mathrm{~F}$ & 27 & China & $\begin{array}{l}\mathrm{CBD} \text { and he- } \\
\text { patic ducts }\end{array}$ & $\begin{array}{l}\text { Painless jaundice } \\
\text { and pruritis }\end{array}$ & $\begin{array}{l}\text { Whipple/resection of } \\
\text { extrahepatic bile duct } \\
\text { and whipple }\end{array}$ & $\begin{array}{l}\text { Villous } \\
\text { adenoma }\end{array}$ & $\begin{array}{l}\text { Well } 9 \text { months after } \\
\text { surgery }\end{array}$ \\
\hline $\begin{array}{l}\text { Saxe et al. } \\
\text { [29] }\end{array}$ & 1 & M & 64 & USA & Distal CBD & $\begin{array}{l}\text { Recurrent abdom- } \\
\text { inal pain, jaun- } \\
\text { dice, weight loss, } \\
\text { pruritis }\end{array}$ & Whipple & $\begin{array}{l}\text { Villous } \\
\text { adenoma }\end{array}$ & Well at 3 years \\
\hline $\begin{array}{l}\text { Blot et al. } \\
{[30]}\end{array}$ & 1 & M & 84 & France & Distal CBD & Febrile jaundice & Surgical excision & $\begin{array}{l}\text { Villous } \\
\text { adenoma }\end{array}$ & Well at 1 year \\
\hline $\begin{array}{l}\text { Inagaki et al. } \\
\text { [31] }\end{array}$ & 1 & M & 73 & Japan & Distal CBD & $\begin{array}{l}\text { Epigastric pain } \\
\text { and jaundice }\end{array}$ & Whipple & $\begin{array}{l}\text { Papillary } \\
\text { adenoma }\end{array}$ & $\begin{array}{l}\text { Well at } 12 \text { months } \\
\text { after surgery }\end{array}$ \\
\hline $\begin{array}{l}\text { Chang et al. } \\
\text { [32] }\end{array}$ & 1 & M & 51 & Taiwan & Distal CBD & $\begin{array}{l}\text { Febrile jaundice, } \\
\text { RUQ pain }\end{array}$ & Refused surgery & $\begin{array}{l}\text { Papillary } \\
\text { adenoma }\end{array}$ & $\begin{array}{l}\text { Asymptomatic after } 3 \\
\text { months }\end{array}$ \\
\hline $\begin{array}{l}\text { Aggarwal et } \\
\text { al. [33] }\end{array}$ & 1 & M & 55 & India & Mid CBD & $\begin{array}{l}\text { Recurrent abdom- } \\
\text { inal pain }\end{array}$ & Whipple & Adenoma & - \\
\hline Lou et al. [34] & 1 & M & 47 & Taiwan & Distal CBD & $\begin{array}{l}\text { Fever, abdominal } \\
\text { pain }\end{array}$ & $\begin{array}{l}\text { Local excision surgi- } \\
\text { cally }\end{array}$ & $\begin{array}{l}\text { Tubular } \\
\text { adenoma }\end{array}$ & Well at 8 months \\
\hline $\begin{array}{l}\text { Fletcher et al. } \\
\text { [35] }\end{array}$ & 1 & M & 74 & UK & Distal CBD & $\begin{array}{l}\text { Painless jaundice, } \\
\text { pruritis, weight } \\
\text { loss }\end{array}$ & Whipple & $\begin{array}{l}\text { Papillary } \\
\text { adenoma }\end{array}$ & $\begin{array}{l}\text { Well at } 1 \text { year after } \\
\text { surgery }\end{array}$ \\
\hline \multirow[t]{3}{*}{ Present cases } & 3 & $\mathrm{~F}$ & 85 & USA & Distal CBD & Abdominal pain & Refused surgery & $\begin{array}{l}\text { Papillary } \\
\text { adenoma }\end{array}$ & $\begin{array}{l}\text { Cholangiocarcinoma } 5 \\
\text { months after presen- } \\
\text { tation }\end{array}$ \\
\hline & & $\mathrm{F}$ & 78 & USA & Distal CBD & $\begin{array}{l}\text { Gallbladder } \\
\text { symptoms }\end{array}$ & $\begin{array}{l}\text { Distal bile duct resec- } \\
\text { tion with choledocho- } \\
\text { jejunostomy }\end{array}$ & Adenoma & $\begin{array}{l}\text { Adenocarcinoma in- } \\
\text { volving small/large } \\
\text { bowel } 4 \text { years after } \\
\text { surgery }\end{array}$ \\
\hline & & $\mathrm{F}$ & 61 & USA & $\begin{array}{l}\text { Common } \\
\text { hepatic } \\
\text { duct }\end{array}$ & $\begin{array}{l}\text { Febrile bactere- } \\
\text { mia }\end{array}$ & $\begin{array}{l}\text { Local excision unsuc- } \\
\text { cessful; total, subse- } \\
\text { quent bile duct resec- } \\
\text { tion and Roux-en-y he- } \\
\text { paticojejunostomy }\end{array}$ & $\begin{array}{l}\text { Villous } \\
\text { adenoma }\end{array}$ & $\begin{array}{l}\text { Metastases to the bone } \\
1 \text { year after initial pre- } \\
\text { sentation }\end{array}$ \\
\hline
\end{tabular}

CBD, common bile duct; CVA, cerebrovascular accident; LFT, liver function test; RUQ, right upper quadrant. 


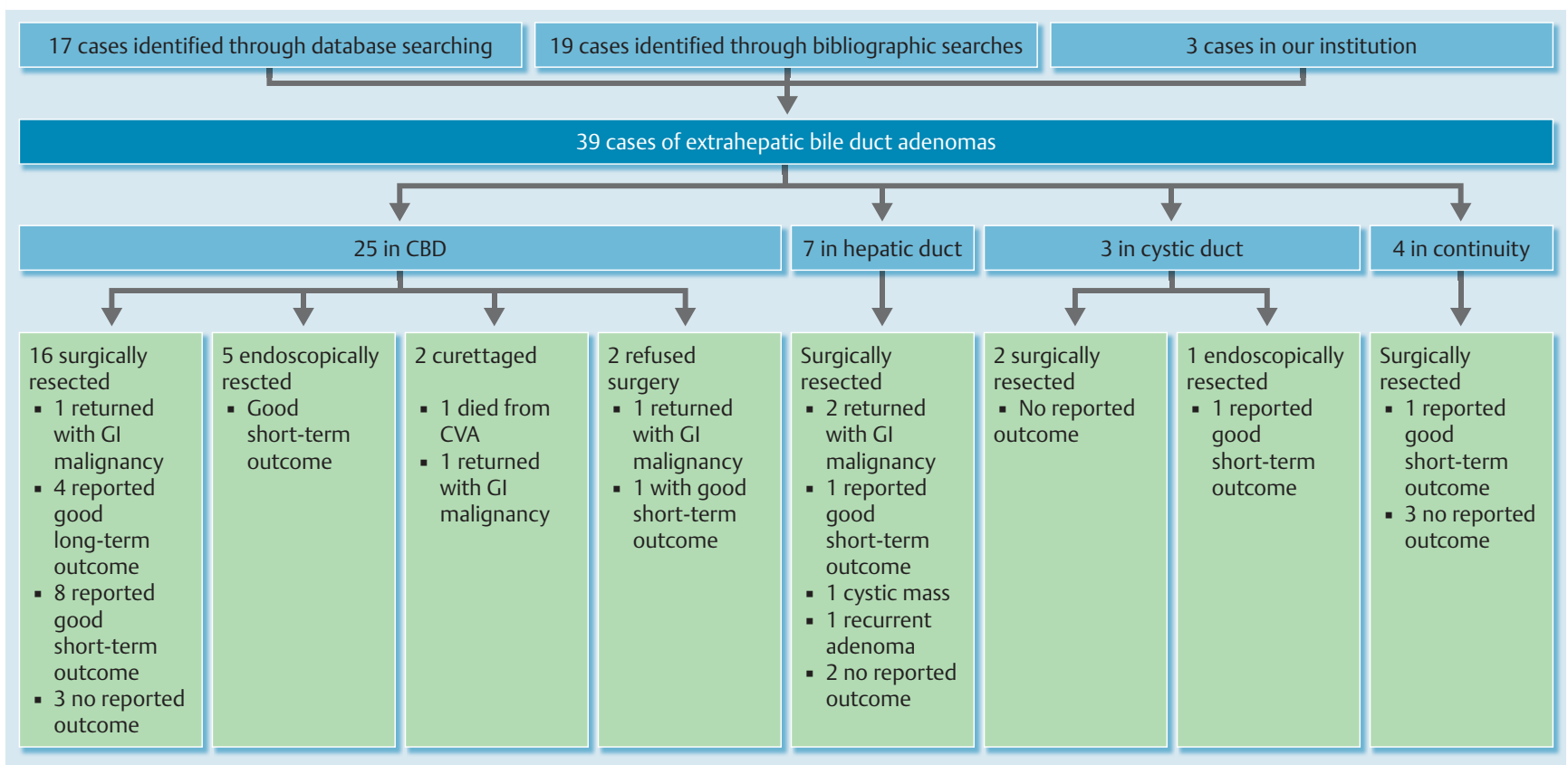

Fig. 4 Flow chart summarizing all 39 reported cases of extrahepatic biliary adenoma.

\section{Discussion \\ $\nabla$}

Benign tumors of the extrahepatic biliary tree can be divided into epithelial and non-epithelial tumors. There is little uniformity in the nomenclature applied to benign epithelial lesions and various classifications have been proposed. According to the WHO classification, they are divided into five different types: tubular, papillary (also known as papillomas), tubulopapillary, biliary cystadenoma, and papillomatosis [1]. Adenomas comprise two-thirds of benign biliary tumors [2]. For the purpose of this review, we have focused on adenomas involving the extrahepatic bile duct, excluding ampullary adenomas, cystadenomas, and papillomatosis. Three extrahepatic bile duct adenomas were diagnosed at our institution among a total of 10559 bile duct pinch biopsies and surgical specimens $(0.03 \%)$ over 13 years. One of our cases has been reported previously [1]. On extensive review of the literature, we found another 36 cases making a total of 39 cases of extrahepatic biliary adenomas reported to date [2-28] ( Table1 and - Fig. 4).

\section{Demographics and presentation}

Extrahepatic biliary adenoma appears to be a disease of older patients. The age of presentation ranged from 15 to 85 years with a mean age of $62.8 \pm 15.4$ years (male, $61.0 \pm 14.4$ years; female, $64.6 \pm 16.3$ years). The affected gender was male in 21 cases $[4,5$, $8,10,11,13,15,16,19,21,25,27]$ and female in 18 cases $[2,3,6,7$, $9,12,14,17,18,20,23,24,26,27]$. The most common presenting complaints were abdominal pain, jaundice, fever, pruritus, and abnormal LFTs. One of our cases presented with recurrent bacteremia in the setting of underlying primary sclerosing cholangitis. Two reported cases were asymptomatic with incidental findings of biliary dilation on imaging $[1,8]$. One case was found incidentally in a surgical resection specimen performed for duodenal adenocarcinoma [11].

\section{Histology}

The pathology specimen was obtained surgically in 32 cases and endoscopically in seven cases. In 22 cases, the adenomas were associated with atypia/dysplasia. The location of adenomas was in the CBD $(25 / 39 ; 64 \%)$ [2-16], common hepatic duct (7/39; $18 \%)[3,17-24]$, and cystic duct (3/39; 8\%) [22-24]. Four (10\%) cases involved multiple ducts in continuity [25-28].

\section{Treatment}

$\nabla$

Management of extrahepatic bile duct adenomas is not clearly defined. Surgical resection was the primary mode of therapy in 31 of 39 patients $[2-5,7,9-13,17-23,25-28]$. Cases in the 1970 s have reported using limited surgical curettage without resection of the affected area [3,5]. Endoscopic resection with snare polypectomy or forceps has been reported in six cases [6, $8,14-16,24]$. There are no reports of the use of ablative therapy with radiofrequency ablation or photodynamic therapy after endoscopic resection.

\section{Prognosis}

The follow-up period varied among all the cases reported. The majority of the patients had good short-term outcomes. Longterm follow-up ( $>1$ year) and short-term outcome ( $<1$ year) were reported in $8[3,7,11,19]$ and 17 cases $[4,5,8,10,11,15-$ $17,21,24,28]$, respectively. Five cases presented with interval malignancy including cholangiocarcinoma, and small-bowel adenocarcinoma was noted at follow-up $[1,4,17]$. The longest followup was reported to be 7 years with the patient still alive [4]. Associations were found with certain malignancies and syndromes either at presentation or follow-up, including Gardner's syndrome, familial polyposis coli, or periampullary carcinoma $[5,7$, 12]. 


\section{Conclusion}

\section{$\nabla$}

We highlight the rarity of extrahepatic bile duct adenoma with three additional cases from our institution adding to the paucity of literature on the subject. All three patients in our series presented with subsequent biliary malignancy with metastases or local invasion. We recommend aggressive surgical intervention and close postoperative surveillance when diagnosis of extrahepatic bile duct adenoma is made.

\section{Competing interests: None}

\section{References}

1 Loh KP, Nautsch D, Desilets $D$ et al. A rare cause of dilated bile duct incidentally detected on imaging. BMJ Case Rep 2014: 2014

2 Ariche A, Shelef I, Hilzenrat $N$ et al. Villous adenoma of the common bile duct transforming into a cholangiocarcinoma. Isr Med Assoc J 2002; 4: $1149-1150$

3 Burhans R, Myers RT. Benign neoplasms of the extrahepatic biliary ducts. Am Surg 1971; 37: 161 - 166

4 Hultén J, Johansson $H$, Olding L. Adenomas of the gallbladder and extrahepatic bile ducts. Acta Chir Scand 1970; 136: 203-207

5 Shemesh E. Adenomatous polyp of the common bile duct in familial polyposis coli. Isr J Med Sci 1985; 21: $701-702$

6 Sturgis TM, Fromkes JJ, Marsh W. Adenoma of the common bile duct: endoscopic diagnosis and resection. Gastrointest Endosc 1992; 38: 504-506

7 Futami H, Furuta T, Hanai H et al. Adenoma of the common human bile duct in Gardner's syndrome may cause relapsing acute pancreatitis. J Gastroenterol 1997; 32: 558-561

8 Jao YTFN, Tseng LJ, Wu CJ et al. Villous adenoma of common bile duct. Gastrointest Endosc 2003; 57: 561 - 562

9 Ibrarullah M, Sreenivasa D. Bile duct adenoma: management by subtotal excision. Trop Gastroenterol Off J Dig Dis Found 2003; 24: 93-94

10 Katsinelos P, Basdanis G, Chatzimavroudis $G$ et al. Pancreatitis complicating mucin-hypersecreting common bile duct adenoma. World J Gastroenterol 2006; 12: 4927-4929

$11 \mathrm{Kim} B S$, Joo SH, Joo KR. Carcinoma in situ arising in a tubulovillous adenoma of the distal common bile duct: a case report. World J Gastroenterol 2008; 14: 4705-4708

12 Aparajita R, Gomez D, Verbeke CS et al. Papillary adenoma of the distal common bile duct associated with a synchronous carcinoma of the peri-ampullary duodenum. JOP 2008; 9: 212-215

13 Akaydin M, Ersoy YE, Erozgen $F$ et al. Tubulovillous adenoma in the common bile duct causing obstructive jaundice. Acta Gastro-Enterol Belg 2009; 72: 450-454

14 Munshi AG, Hassan MA. Common bile duct adenoma: case report and brief review of literature. Surg Laparosc Endosc Percutan Tech 2010; 20: e193-194
15 Prachayakul V, Aswakul P, Kachintorn U. Incidental removal of distal common bile duct adenoma after plastic stent placement. Endoscopy 2012; 44: 02 UCTN E11-12

16 Sirimontaporn N, Aswakul P, Junyangdikul P et al. Early neoplasia of the common bile duct diagnosed and completely removed using multiple endoscopic modalities. Endoscopy 2013; 45: 02 UCTN E102-103

17 Styne P, Warren GH, Kumpe DA et al. Obstructive cholangitis secondary to mucus secreted by a solitary papillary bile duct tumor. Gastroenterology 1986; 90: $748-753$

18 Cardoza J, Schrumpf J, Skioldebrand C et al. Biliary obstruction caused by a papilloma of the common hepatic duct. J Ultrasound Med Off J Am Inst Ultrasound Med 1988; 7: 467-469

19 Jennings PE, Rode J, Coral $A$ et al. Villous adenoma of the common hepatic duct: the role of ultrasound in management. Gut 1990; 31: 558 560

20 Colarian JH, Wescott CJ. Villous adenoma of the common hepatic duct. Gastrointest Endosc 2001; 54: 226

21 Sotona 0 , Cecka F, Neoral C et al. Papillary adenoma of the extrahepatic biliary tract - a rare cause of obstructive jaundice. Acta Gastro-Enterol Belg 2010; 73: 270-273

22 Ho C-M, Lee P-H. Image of the month. Papillary adenoma of the cystic duct. Arch Surg Chic Ill 1960 2006; 141: 315

23 Loh A, Kamar S, Dickson GH. Solitary benign papilloma (papillary adenoma) of the cystic duct: a rare cause of biliary colic. Br J Clin Pract 1994; 48: $167-168$

24 Liu Z, Lv C, Cui G et al. Gastroscopic snare polypectomy for cystic duct adenoma: a rare occurrence. Endoscopy 2014; 46: 01 UCTN E143145

25 O'Shea M, Fletcher HS, Lara JF. Villous adenoma of the extrahepatic biliary tract: a rare entity. Am Surg 2002; 68: 889-891

26 Morris-Stiff GJ, Senda Y, Verbeke CS. Papillary adenoma arising in the left hepatic duct: an unusual tumour in an uncommon location. Eur J Gastroenterol Hepatol 2010; 22: 886-888

27 Hanafy M, McDonald P. Villous adenoma of the common bile duct. J R Soc Med 1993; 86: 603-604

$28 \mathrm{Xu} H X$, Chen $L D$. Villous adenoma of extrahepatic bile duct: Contrastenhanced sonography findings. J Clin Ultrasound 2008; 36: 39-41

29 Saxe J, Lucas C, Ledgerwood AM et al. Villous adenoma of the common bile duct. Arch Surg Chic Ill 1960 1988; 123: 96

30 Blot E, Heron F, Cardot $F$ et al. Villous adenoma of the common bile duct. J Clin Gastroenterol 1996; 22: 77-79

31 Inagaki M, Ishizaki A, Kino $S$ et al. Papillary adenoma of the distal common bile duct. J Gastroenterol 1999; 34: 535-539

32 Chang YT, Wang HP, Sun CT et al. Papillary adenoma of the bile duct. Gastrointest Endosc 2001; 53: 777

33 Aggarwal S, Kumar S, Kumar A et al. Extra-hepatic bile duct adenoma in a patient with a choledochal cyst. J Gastroenterol Hepatol 2003; 18: $351-352$

34 Lou HY, Chang CC, Chen SH et al. Acute cholangitis secondary to a common bile duct adenoma. Hepatogastroenterology 2003; 50: 949-951

35 Fletcher ND, Wise PE, Sharp KW. Common bile duct papillary adenoma causing obstructive jaundice: case report and review of the literature. Am Surg 2004; 70: 448-452 